\title{
Downregulation of long non-coding RNA LINC-PINT serves as a diagnostic and prognostic biomarker in patients with non-small cell lung cancer
}

\author{
CHUNJIE ZHANG ${ }^{1}$, CUIXUE GONG ${ }^{2}$, JIANZHAO LI $^{3}$ and JIAYING TANG ${ }^{4}$ \\ ${ }^{1}$ Department of Clinical Laboratory, ${ }^{2}$ Outpatient Dressing Room, Departments of ${ }^{3}$ Pathology and ${ }^{4}$ Blood Transfusion, \\ Zibo Central Hospital, Zibo, Shandong 255036, P.R. China
}

Received August 19, 2020; Accepted December 23, 2020

DOI: $10.3892 / \mathrm{ol} .2021 .12471$

\begin{abstract}
Long non-coding RNAs (lncRNAs) play an important role in gene regulation. Several lncRNAs have been demonstrated to be associated with the diagnosis and prognosis of non-small cell lung cancer (NSCLC). The present study aimed to investigate the role of lncRNA long intragenic non-protein-coding RNA p53-induced transcript (LINC-PINT) in NSCLC to identify a novel non-invasive biomarker for the diagnosis and prognosis of patients with NSCLC. Reverse transcription-quantitative PCR analysis was performed to detect LINC-PINT expression in the tissue and serum samples of patients with NSCLC. The diagnostic and prognostic values of LINC-PINT were assessed via the receiver operating characteristic curve, and Kaplan-Meier and Cox regression analyses, respectively. The results demonstrated that LINC-PINT expression was significantly downregulated in NSCLC serum samples and tissues. In addition, serum LINC-PINT exhibited diagnostic value in patients with NSCLC, and may be used to predict prognosis. Furthermore, aberrant LINC-PINT expression in tumor tissues was significantly associated with lymph node metastasis, tumor size, differentiation and TNM stage. Taken together, the results of the present study suggest that lncRNA LINC-PINT may be an independent diagnostic and prognostic biomarker in NSCLC.
\end{abstract}

\section{Introduction}

Lung cancer is the most common cause of cancer-associated mortality worldwide $(1,2)$. The 10 -year survival rate of patients following diagnosis across all stages of lung cancer is $<7 \%$ (3).

Correspondence to: Dr Jiaying Tang, Department of Blood Transfusion, Zibo Central Hospital, 54 Gongqingtuan Road, Zibo, Shandong 255036, P.R. China

E-mail: tangjy_zch@163.com

Key words: non-small cell lung cancer, long intragenic non-protein-coding RNA p53-induced transcript, diagnostic, prognostic
Despite advancements in diagnosis, classification and therapy, the overall survival rate of patients with lung cancer remains poor (4). Non-small cell lung cancer (NSCLC) accounts for $85 \%$ of all lung cancers (5). Patients with advanced or metastatic NSCLC have poor survival outcomes, thus highlighting the need for more effective therapies (6). Although the diagnosis and treatment of NSCLC are continuously being improved, patient prognosis remains unfavorable (7). Currently, the 5 -year overall survival rate is only $15 \%$ (8). Thus, it remains critical to identify novel effective biomarkers for accurate early diagnosis and improved prognosis of patients with NSCLC.

Long non-coding RNAs (lncRNAs) are a novel class of non-coding RNAs, usually defined as RNA molecules $>200$ nucleotides in length (9). IncRNAs function as major regulators for gene expression, and thus play key roles in several biological functions and disease processes, including cancer $(10,11)$. The lncRNA, long intragenic non-protein-coding RNA p53-induced transcript (LINC-PINT), is abnormally expressed in several tumors, including gastric cancer, renal cell carcinoma and glioblastoma, and exhibits certain diagnostic and prognostic values (12-15). In NSCLC, LINC-PINT has been demonstrated to act as a tumor suppressor by sponging microRNA (miRNA/miR)-208a-3p and regulating programmed cell death 4 (PDCD4) (16). Wang et al (17) reported that LINC-PINT plays an important role in NSCLC by sponging miR-543 and inducing PTEN. However, the clinical value of LINC-PINT in the diagnosis and prognosis of NSCLC remains unclear.

Thus, the present study aimed to investigate the clinical significance of LINC-PINT in patients with NSCLC. The diagnostic and prognostic values of LINC-PINT were also assessed via the receiver operating characteristic (ROC) curve, and Kaplan-Meier and Cox regression analyses.

\section{Materials and methods}

Patients and tissue collection. A total of 122 patients who were pathologically diagnosed with NSCLC and received resection surgery between March 2011 and June 2014 in Zibo Central Hospital were enrolled in the present study. The patients included 53 women and 69 men with a mean age of $61.7 \pm 13.2$ years (age range, $38-84$ years old). All patients were included following the inclusion criteria: i) Tumor tissues 
were histopathologically diagnosed with NSCLC; ii) Cases had complete demographic and clinical data; iii) Cases signed informed consent for the use of clinical samples and data. In addition, the exclusion criteria for patient recruitment were as follows: i) Patients with a history of other types of cancer; ii) Cases aged $<18$ years; iii) Pregnant or lactating women; iv) Cases received preoperative antitumor therapy. In addition, 62 age (mean age, $60.8 \pm 13.8$ years; age range, $37-82$ years) and sex ( 25 women and 37 men) matched healthy individuals willing to participate in the present study during this period were enrolled to serve as controls. Blood samples were collected from all participants and immediately centrifuged at $1,500 \mathrm{x}$ for $10 \mathrm{~min}$ at $4^{\circ} \mathrm{C}$ for serum extraction.

NSCLC tissues and adjacent normal tissues (at least $3 \mathrm{~cm}$ from the edge of tumor) were extracted from the patients during resection surgery and frozen in liquid nitrogen at $-80^{\circ} \mathrm{C}$. Demographic and clinicopathological characteristics, and the 5-year follow-up survey (range, 0-60 months), monthly phone calls were made for each patient and collected survival information of the patients for subsequent analyses. Cases that died from external events were excluded. The present study was approved the Ethics Committee of Zibo Central Hospital (Zibo, China; approval no. ZCHh-110824), and written informed consent was provided by all participants prior to the study start.

Bioinformatics analysis based on The Cancer Genome Atlas (TCGA) database. LINC-PINT expression in NSCLC and its association with survival prognosis was assessed using the Gene Expression Profiling Interactive Analysis (GEPIA) database (http://gepia.cancer-pku.cn/index.html) (18), based on TCGA database (https://cancergenome.nih.gov).

Reverse transcription-quantitative $(R T-q) P C R$. Total RNA was extracted from fresh tissue and serum samples using the GenElute Total RNA Purification kit (Sigma-Aldrich; Merck KGaA; cat. no. RNB100). The concentration and quality were assessed using the NanoDrop 2000 (Thermo Fisher Scientific, Inc.), in which RNA with an absorbance ratio of optical density (OD) 260/OD 280 results close to 2.0 were used for subsequent RT. RT was performed using the Applied Biosystems High-Capacity cDNA Reverse Transcription kit (Thermo Fisher Scientific, Inc.; cat. no. 43-688-13), and the resulting cDNA was stored at $-20^{\circ} \mathrm{C}$. cDNA was subsequently used as the template for qPCR, which was performed using the SYBR-Green I Master Mix kit (Invitrogen; Thermo Fisher Scientific, Inc.; cat. no. 4334973) and the 7500 Real-Time PCR System (Applied Biosystems; Thermo Fisher Scientific, Inc.). The following thermocycling conditions were used: $95^{\circ} \mathrm{C}$ for $10 \mathrm{~min}$, followed by 40 cycles of $95^{\circ} \mathrm{C}$ for $30 \mathrm{sec}, 58^{\circ} \mathrm{C}$ for $20 \mathrm{sec}$ and $72^{\circ} \mathrm{C}$ for $30 \mathrm{sec}$. The primer sequences were as follows: LINC-PINT forward, 5'-CGTGGGAGCCCCTTT AAGTT-3' and reverse, 5'-GGGAGGTGGCGTAGTTTC TC-3'; GAPDH forward 5'-CCTCTGACTTCAACAGCG ACAC-3' and reverse, 5'-TGGTCCAGGGGTCTTACTCC-3'. Relative expression levels were calculated using the $2^{-\Delta \Delta C a}$ method (19) and normalized to the internal reference gene GAPDH. Each analysis was repeated at least three times.

Statistical analysis. Statistical analysis was performed using SPSS 21.0 software (IBM Corp.) and GraphPad 7.0 software
(GraphPad Software, Inc.). Data are presented as the mean \pm standard deviation. Paired Student's t-test was used to compare the difference in LINC-PINT expression between NSCLC tissues and adjacent normal tissues, while unpaired Student's t-test was used to compare serum LINC-PINT expression between patients with NSCLC and healthy individuals, and perform expression analysis of LINC-PINT using the GEPIA database. The $\chi^{2}$ test was used to assess the association between LINC-PINT expression and the clinicopathological characteristics of patients with NSCLC. ROC curves were used to determine the diagnostic value of LINC-PINT, while Kaplan-Meier and Cox regression analyses were performed to determine the prognostic value of LINC-PINT in NSCLC. $\mathrm{P}<0.05$ was considered to indicate a statistically significant difference.

\section{Results}

LINC-PINT expression in NSCLC based on TCGA database. Data mining TCGA database using the GEPIA database demonstrated that LINC-PINT expression is significantly downregulated in NSCLC tissues compared with normal tissue $(\mathrm{P}<0.05$; Fig. 1A). Kaplan-Meier survival analysis demonstrated that patients with low LINC-PINT expression had a shorter overall survival time than those with high LINC-PINT expression (Fig. 1B). In addition, the survival curve plotted by GEPIA demonstrated that low LINC-PINT expression was significantly associated with poor prognosis of patients with NSCLC $(\mathrm{P}=0.00084)$.

LINC-PINT expression in NSCLC. To further determine the role of LINC-PINT in NSCLC, RT-qPCR analysis was performed to detect LINC-PINT expression in NSCLC tissue and serum samples. The results demonstrated that serum LINC-PINT expression was significantly downregulated in patients with NSCLC compared with the healthy individuals $(\mathrm{P}<0.001$; Fig. 2A). Similarly, LINC-PINT expression was significantly downregulated in NSCLC tissues compared with adjacent normal tissues $(\mathrm{P}<0.001$; Fig. $2 \mathrm{~B})$. These experimental results are consistent with the analysis results from TCGA database.

Diagnostic value of serum LINC-PINT in patients with NSCLC. The diagnostic value of LINC-PINT in patients with NSCLC was assessed. A ROC curve was established (Fig. 3), which demonstrated that LINC-PINT had high diagnostic value, with an area under the curve (AUC) value of 0.873 , sensitivity of $90.9 \%$ and specificity of $75.8 \%$. The ideal cut-off value was 1.236 .

Association between LINC-PINT expression and the clinicopathological characteristics of patients with NSCLC. As presented in Table I, LINC-PINT expression was significantly associated with lymph node metastasis $(\mathrm{P}=0.019)$, differentiation $(\mathrm{P}=0.028)$, tumor-node-metastasis (TNM) stage $(20)(\mathrm{P}=0.020)$ and tumor size $(\mathrm{P}=0.027)$. Conversely, no significant associations were observed between LINC-PINT expression and age, sex and smoking history (all $\mathrm{P}>0.05$ ).

Prognostic value of LINC-PINT in patients with NSCLC. Due to the ectopic expression of LINC-PINT in NSCLC (16), its prognostic value in patients with NSCLC was assessed. 

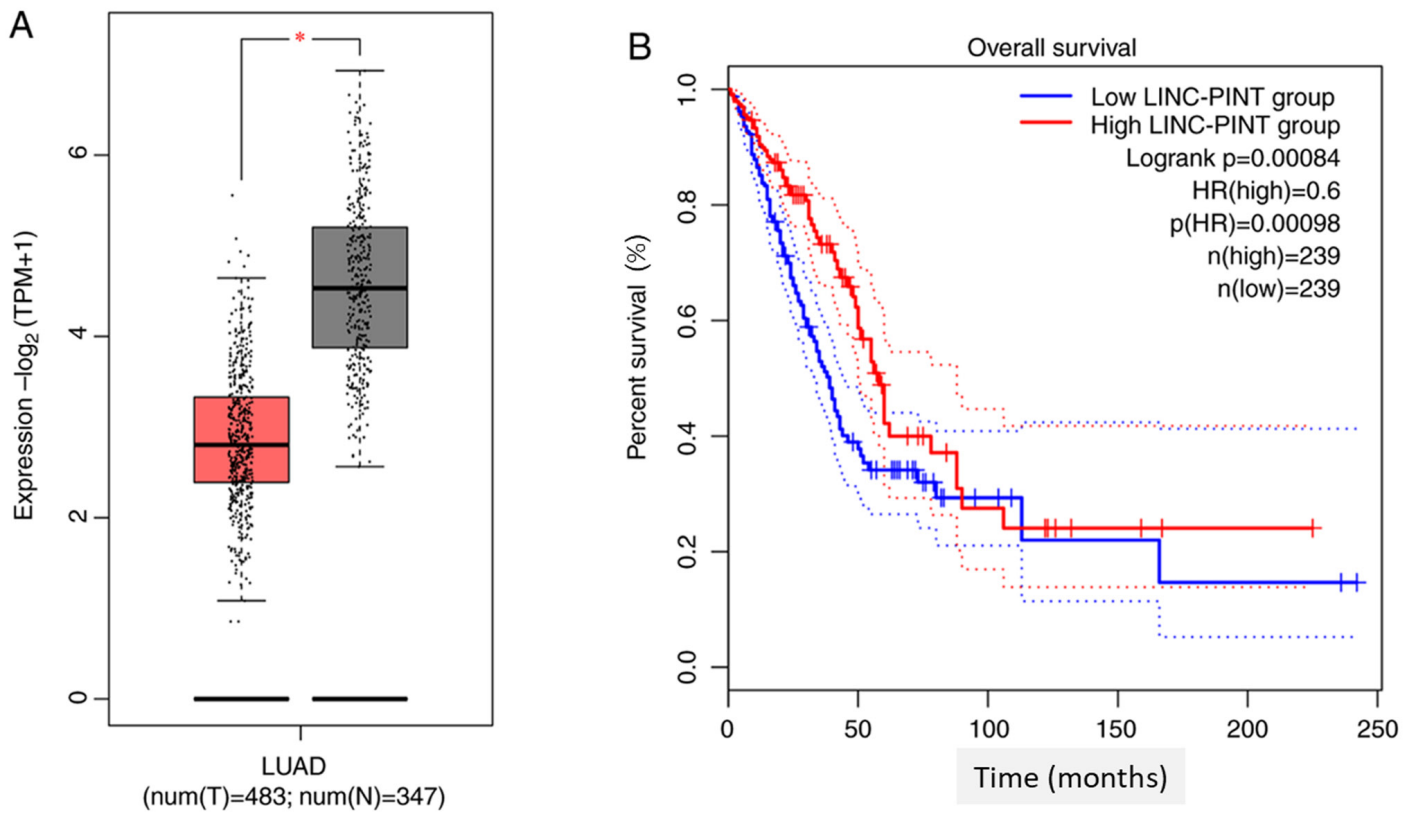

Figure 1. LINC-PINT expression in NSCLC based on The Cancer Genome Atlas database. (A) LINC-PINT expression in NSCLC tissues and normal tissues. (B) Survival analysis of patients with NSCLC, with different expression levels of LINC-PINT. "P<0.05. LINC-PINT, long intragenic non-protein-coding RNA p53-induced transcript; NSCLC, non-small cell lung cancer; T, tumor; N, normal.
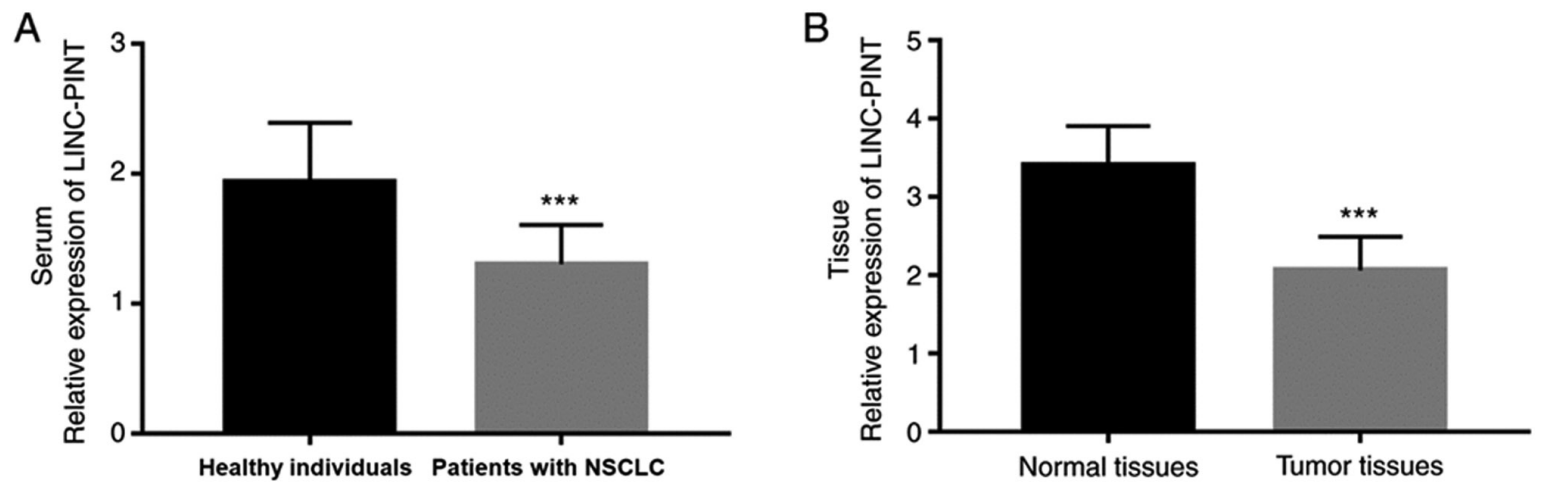

Figure 2. LINC-PINT expression in NSCLC. (A) Serum LINC-PINT expression was significantly downregulated in patients with NSCLC compared with the healthy individuals. (B) LINC-PINT expression was significantly downregulated in NSCLC tissues compared with adjacent normal tissues. ${ }^{* * *} \mathrm{P}<0.001$. LINC-PINT, long intragenic non-protein-coding RNA p53-induced transcript; NSCLC, non-small cell lung cancer.

Kaplan-Meier survival analysis was performed to assess the association between LINC-PINT expression and overall survival of patients with NSCLC (Fig. 4). The results demonstrated that patients with high LINC-PINT expression had a significantly longer overall survival time than those with low LINC-PINT expression $(\mathrm{P}=0.002)$. Furthermore, the univariate and multivariate Cox regression analysis demonstrated that LINC-PINT [hazard ratio (HR), 2.628; 95\% confidence interval $(\mathrm{CI}), 1.589-4.348 ; \mathrm{P}<0.001]$ and $\mathrm{TNM}$ stage (HR, 1.810; 95\% CI, 1.091-3.004; $\mathrm{P}=0.022$ ) were two independent prognostic factors for the survival of patients with NSCLC (Table II).

\section{Discussion}

Lung cancer is the most common malignant tumor worldwide, with the highest mortality rate $(17,21)$. NSCLC is the main type of lung cancer, which accounts for $\sim 85 \%$ of all lung cancer cases (22), and $\sim 30 \%$ of patients have metastatic disease at diagnosis (23). NSCLC has slower proliferation and division of cancer cells, and relatively late spread and metastasis compared with small cell carcinoma (24). Thus, despite advancements in treatment, the prognosis of patients with NSCLC remains poor, and the 5-year overall survival rate does not exceed $16 \%$ (25). Accurate biomarkers are useful in predicting the diagnosis and prognosis of different diseases, including NSCLC. Previous studies have proposed several biomarkers for NSCLC (26-28). Among these, lncRNAs offer a new direction and have attracted notable attention.

Several types of lncRNAs have been studied in NSCLC. For example, Zhang et al (29) demonstrated that lncRNA FENDRR inhibits the progression of NSCLC by binding to miR-761 and regulating TIMP2 expression. In addition, lncRNA FEZF1-AS1 can act as a tumor promoting regulator in NSCLC and may provide a target for the treatment of NSCLC (30). It has been demonstrated that MALAT1 
Table I. Association between LINC-PINT expression and the clinicopathological characteristics of patients with non-small cell lung cancer $(\mathrm{n}=122)$.

LINC-PINT expression

Characteristic $\quad$ Number of patients, $n \quad$ Low $(\mathrm{n}=64) \quad$ High $(\mathrm{n}=58) \quad$ P-value

Age, years

$\leq 60$

$>60$

Sex

Female

Male

Smoking history

Never

Ever

Tumor size, $\mathrm{cm}$

$\leq 3$

$>3$

Differentiation

Well/moderate

Poor

Lymph node metastasis

Negative

Positive

TNM stage

I-II

III-IV
46

76

53

69

52

70

65

57

63

59

60

62

56

66
24

40

28

36

26

38

28

36

27

37

25

39

23

41
0.961

22

36

25

33

26

32

37

21

36

22

35

23

TNM, tumor-node-metastasis.

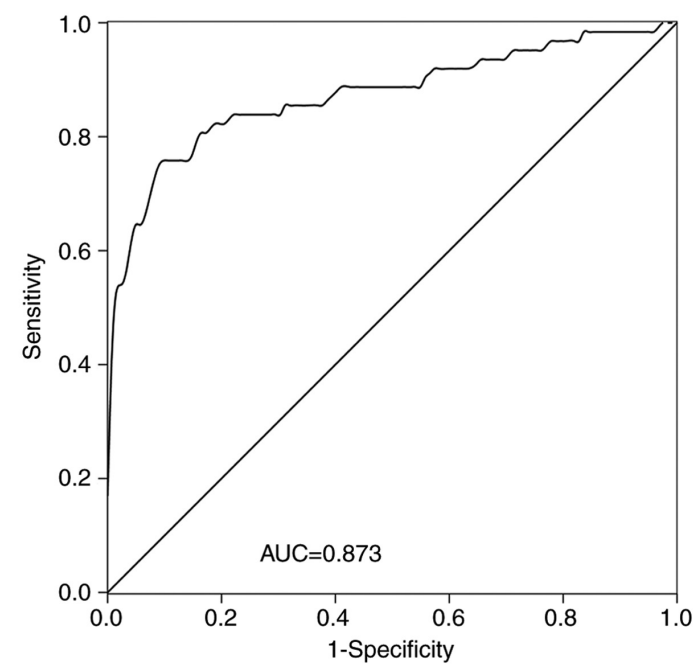

Figure 3. Diagnostic value of LINC-PINT in NSCLC. Receiver operating characteristic curve for patients with NSCLC, based on LINC-PINT expression. LINC-PINT, long intragenic non-protein-coding RNA p53-induced transcript; NSCLC, non-small cell lung cancer; AUC, area under the curve.

can alter chemoresistance of NSCLC cells by targeting miR-197-3p and regulating p120-ctn expression, which may assist in improving chemotherapies for NSCLC (31).

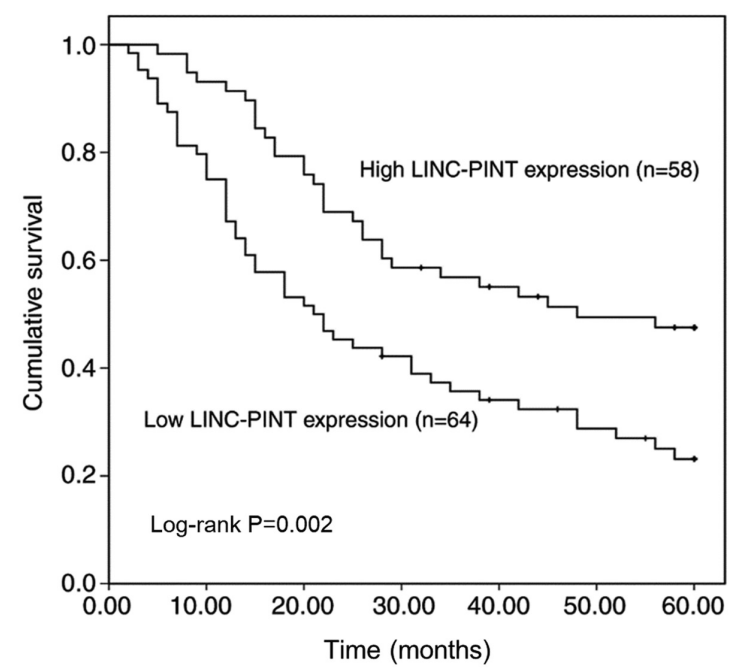

Figure 4. Prognostic value of LINC-PINT in NSCLC. Kaplan-Meier survival analysis for patients with NSCLC, based on LINC-PINT expression. LINC-PINT, long intragenic non-protein-coding RNA p53-induced transcript; NSCLC, non-small cell lung cancer.

Collectively, these results suggest that lncRNAs play important roles in the development and progression of NSCLC. Recently, IncRNA LINC-PINT has been extensively studied. 
Table II. Cox regression analysis of patients with non-small cell lung cancer.

\begin{tabular}{|c|c|c|c|c|c|c|}
\hline \multirow[b]{2}{*}{ Variable } & \multicolumn{3}{|c|}{ Univariate analysis } & \multicolumn{3}{|c|}{ Multivariate analysis } \\
\hline & $\mathrm{HR}$ & $95 \% \mathrm{CI}$ & P-value & HR & $95 \% \mathrm{CI}$ & P-value \\
\hline LINC-PINT & 2.845 & $1.629-4.555$ & $<0.001$ & 2.628 & $1.589-4.348$ & $<0.001$ \\
\hline Age, years & 1.141 & $0.761-1.674$ & 0.499 & 1.167 & $0.721-1.888$ & 0.529 \\
\hline Sex & 1.411 & $0.857-2.166$ & 0.285 & 1.479 & $0.915-2.390$ & 0.110 \\
\hline Smoking & 1.418 & $0.869-2.087$ & 0.221 & 1.323 & $0.833-2.102$ & 0.236 \\
\hline Tumor size & 1.396 & $0.925-1.968$ & 0.104 & 1.146 & $0.714-1.838$ & 0.573 \\
\hline Differentiation & 1.401 & $0.991-2.120$ & 0.059 & 1.358 & $0.832-2.218$ & 0.221 \\
\hline Lymph node metastasis & 1.446 & $1.089-2.047$ & 0.037 & 1.316 & $0.819-2.115$ & 0.257 \\
\hline TNM stage & 2.041 & $1.351-3.184$ & 0.010 & 1.810 & $1.091-3.004$ & 0.022 \\
\hline
\end{tabular}

TNM, tumor-node-metastasis; HR, hazard ratio; CI, confidence interval.

It has been suggested that LINC-PINT may mediate cancer cell proliferation, invasion and migration in osteosarcoma by binding to miRNA-21 (32). Furthermore, Zhang et al (16) demonstrated that LINC-PINT mediates inhibition of cell proliferation, cell cycle, and cell migration and invasion in NSCLC via the miR-208a-3p/PDCD4 axis. However, the clinicopathological characteristics of LINC-PINT in NSCLC remain unclear.

In the present study, TCGA data mining and RT-qPCR analyses demonstrated that LINC-PINT expression was significantly downregulated in NSCLC tissues compared with normal tissues, which was consistent with the findings by Wang et al (17). Thus, it was predicted that LINC-PINT may be involved in the progression of NSCLC. To further investigate its role in the development of NSCLC, the association between LINC-PINT expression and the clinicopathological characteristics of patients with NSCLC was assessed. The results demonstrated that LINC-PINT expression in NSCLC was significantly associated with lymph node metastasis, differentiation, TNM stage and tumor size.

The clinical significance of LINC-PINT in NSCLC was further investigated. The results demonstrated that abnormal LINC-PINT expression was associated with the diagnosis or prognosis of patients with NSCLC. IncRNAs are considered ideal diagnostic tools for different human diseases due to their specific expression and stability in blood samples (11). For example, decreased serum lncRNA-D16366 levels serve as a non-invasive diagnostic biomarker in patients with hepatocellular carcinoma (33), and enhanced serum lncRNA-XLOC_009167 levels may serve as a biomarker for the diagnosis of patients with lung cancer (34). The results of the present study demonstrated that downregulated LINC-PINT expression increased diagnostic accuracy in patients with NSCLC. Previous studies have investigated the diagnostic value of some lncRNAs and a study by Xie et al (35), which investigated circulating IncRNAs for NSCLC diagnosis, reported that SOX2OT, ANRIL, CEA, CYFRA211 and SCCA may serve as candidate diagnostic biomarkers. In addition, the combined diagnostic accuracy of the lncRNAs exhibited an AUC value of 0.853 . The results of the present study demonstrated that the AUC value of LINC-PINT was 0.873, suggesting that LINC-PINT may be a potential diagnostic biomarker for patients with NSCLC. The prognostic value of LINC-PINT in NSCLC was also assessed in the present study. Cancer prognosis relies on the TNM system, which requires medical imaging support such as $\mathrm{CT}$, magnetic resonance and bone scan (36). The TNM method not only consumes manpower and material resources, but also has a long-time cycle (37), thus, there is an urgent requirement to identify and develop novel prognostic biomarkers. IncRNAs have been used as biomarkers in different types of cancer (38). In the present study, the prognostic value of LINC-PINT was assessed based on the 5-year follow-up survival information of patients with NSCLC. Kaplan-Meier survival analysis demonstrated that patients with low LINC-PINT expression had a shorter overall survival time than those with high LINC-PINT expression. In addition, multivariate Cox regression analysis confirmed that LINC-PINT expression can effectively be used to predict the prognosis of patients with NSCLC.

The biological function of LINC-PINT has been investigated in NSCLC progression. For example, Wang et al (17) demonstrated that LINC-PINT can inhibit the cell proliferation and cell colony formation of NSCLC cells, and it was concluded that LINC-PINT plays an important biological role in NSCLC by sponging miR-543 and inducing PTEN expression. Although this study provides evidence for the clinical value of LINC-PINT in the diagnosis and prognosis of patients with NSCLC, the miRNA that may be regulated by LINC-PINT in NSCLC was not investigated in the present study. Considering the regulatory association between LINC-PINT and miRNA in NSCLC, the clinical significance of LINC-PINT may be improved by co-analyzing the expression changes in the miRNAs. Thus, further studies are required to confirm and develop the clinical application potential of LINC-PINT, with a larger study population and analyses of related miRNAs.

In conclusion, the results of the present study demonstrated that lncRNA LINC-PINT expression is downregulated in NSCLC tissue and serum samples. Furthermore, serum LINC-PINT may serve as a candidate diagnostic biomarker 
to distinguish patients with NSCLC from healthy individuals, and low LINC-PINT expression in tumor tissues may predict poor prognosis of patients with NSCLC.

\section{Acknowledgements}

Not applicable.

\section{Funding}

No funding was received.

\section{Availability of data and materials}

All data generated or analyzed during the present study are included in this published article.

\section{Authors' contributions}

$\mathrm{CZ}$ and JT contributed to the conception of the work, bioinformatics analysis, data analysis and interpretation, manuscript writing and revision, and confirmed the authenticity of all the raw data. CG and JL collected the clinical samples and data and performed the experiments. All authors have read and approved the final manuscript.

\section{Ethics approval and consent to participate}

The present study was approved the Ethics Committee of Zibo Central Hospital (Zibo, China; approval no. ZCHh-110824), and written informed consent was provided by all participants prior to the study start.

\section{Patient consent for publication}

Not applicable.

\section{Competing interests}

The authors declare that they have no competing interests.

\section{References}

1. Nanavaty P, Alvarez MS and Alberts WM: Lung cancer screening: Advantages, controversies, and applications. Cancer Control 21: 9-14, 2014.

2. Nasim F, Sabath BF and Eapen GA: Lung cancer. Med Clin North Am 103: 463-473, 2019.

3. Cheung CHY and Juan HF: Quantitative proteomics in lung cancer. J Biomed Sci 24: 37, 2017.

4. de Sousa VML and Carvalho L: Heterogeneity in lung cancer. Pathobiology 85: 96-107, 2018

5. Herbst RS, Morgensztern D and Boshoff C: The biology and management of non-small cell lung cancer. Nature 553: 446-454, 2018.

6. Syrigos KN, Saif MW, Karapanagiotou EM, Oikonomopoulos G and De Marinis F: The need for third-line treatment in non-small cell lung cancer: An overview of new options. Anticancer Res 31: 649-659, 2011.

7. Richard PJ and Rengan R: Oligometastatic non-small-cell lung cancer: Current treatment strategies. Lung Cancer (Auckl) 7: 129-140, 2016.

8. Hirsch FR, Suda K, Wiens J and Bunn PA Jr: New and emerging targeted treatments in advanced non-small-cell lung cancer. Lancet 388: 1012-1024, 2016.
9. Wang J, Su Z, Lu S, Fu W, Liu Z, Jiang X and Ta S: LncRNA HOXA-AS2 and its molecular mechanisms in human cancer. Clin Chim Acta 485: 229-233, 2018.

10. Peng WX, Koirala P and Mo YY: LncRNA-Mediated regulation of cell signaling in cancer. Oncogene 36: 5661-5667, 2017.

11. Bhan A, Soleimani M and Mandal SS: Long noncoding RNA and cancer: A new paradigm. Cancer Res 77: 3965-3981, 2017.

12. Feng $\mathrm{H}$, Zhang J, Shi Y, Wang L, Zhang $\mathrm{C}$ and Wu L: Long noncoding RNA LINC-PINT is inhibited in gastric cancer and predicts poor survival. J Cell Biochem 120: 9594-9600, 2019.

13. Duan J, Ma X, Shi J, Xuan Y, Wang H, Li P, Zhang Y, Fan Y, Gong H, Ma X, et al: Long noncoding RNA LINC-PINT promotes proliferation through EZH2 and predicts poor prognosis in clear cell renal cell carcinoma. Onco Targets Ther 12: 4729-4740, 2019.

14. Hong L, Wang H, Wang J, Wei S, Zhang F, Han J, Liu Y, Ma M, Liu C, Xu Y and Jiang D: LncRNA PTCSC3 inhibits tumor growth and cancer cell stemness in gastric cancer by interacting with lncRNA linc-pint. Cancer Manag Res 11: 10393-10399. 2019.

15. Zhang M, Zhao K, Xu X, Yang Y, Yan S, Wei P, Liu H, Xu J, Xiao F, Zhou H, et al: A peptide encoded by circular form of LINC-PINT suppresses oncogenic transcriptional elongation in glioblastoma. Nat Commun 9: 4475, 2018.

16. Zhang L, Hu J, Li J, Yang Q, Hao M and Bu L: Long noncoding RNA LINC-PINT inhibits non-small cell lung cancer progression through sponging miR-218-5p/PDCD4. Artif Cells Nanomed Biotechnol 47: 1595-1602, 2019.

17. Wang S, Jiang W, Zhang X, Lu Z, Geng Q, Wang W, Li N and Cai X: LINC-PINT alleviates lung cancer progression via sponging miR-543 and inducing PTEN. Cancer Med 9: 1999-2009, 2020.

18. Tang Z, Li C, Kang B, Gao G, Li C and Zhang Z: GEPIA: A web server for cancer and normal gene expression profiling and interactive analyses. Nucleic Acids Res 45: W98-W102, 2017.

19. Livak KJ and Schmittgen TD: Analysis of relative gene expression data using real-time quantitative PCR and the 2(-Delta Delta C(T)) method. Methods 25: 402-408, 2001.

20. Chassagnon G, Bennani S and Revel MP: New TNM classification of non-small cell lung cancer. Rev Pneumol Clin 73: 34-39, 2017 (In French).

21. Collins LG, Haines C, Perkel R and Enck RE: Lung cancer: Diagnosis and management. Am Fam Physician 75: 56-63, 2007.

22. Ettinger DS, Wood DE, Aisner DL, Akerley W, Bauman J, Chirieac LR, D'Amico TA, DeCamp MM, Dilling TJ, Dobelbower M, et al: Non-Small cell lung cancer, version 5.2017, NCCN clinical practice guidelines in oncology. J Natl Compr Cancer Netw 15: 504-535, 2017.

23. Gong HY, Wang Y, Han G and Song QB: Radiotherapy for oligometastatic tumor improved the prognosis of patients with non-small cell lung cancer (NSCLC). Thorac Cancer 10: 1136-1140, 2019.

24. Jonna S and Subramaniam DS: Molecular diagnostics and targeted therapies in non-small cell lung cancer (NSCLC): An update. Discov Med 27: 167-170, 2019.

25. Chen G, Umelo IA, Lv S, Teugels E, Fostier K, Kronenberger P, Dewaele A, Sadones J, Geers C and De Grève J: MiR-146a inhibits cell growth, cell migration and induces apoptosis in non-small cell lung cancer cells. PLoS One 8: e60317, 2013.

26. Camidge DR, Doebele RC and Kerr KM: Comparing and contrasting predictive biomarkers for immunotherapy and targeted therapy of NSCLC. Nat Rev Clin Oncol 16: 341-355, 2019.

27. Gao P, Wang H, Yu J, Zhang J, Yang Z, Liu M, Niu Y, Wei X, Wang W, Li H, et al: MiR-3607-3p suppresses non-small cell lung cancer (NSCLC) by targeting TGFBR1 and CCNE2. PLoS Genet 14: e1007790, 2018.

28. Wang SY, Li Y, Jiang YS and Li RZ: Investigation of serum miR-411 as a diagnosis and prognosis biomarker for non-small cell lung cancer. Eur Rev Med Pharmacol Sci 21: 4092-4097, 2017.

29. Zhang G, Wang Q, Zhang X, Ding Z and Liu R: LncRNA FENDRR suppresses the progression of NSCLC via regulating miR-761/TIMP2 axis. Biomed Pharmacother 118: 109309, 2019.

30. He R, Zhang FH and Shen N: LncRNA FEZF1-AS1 enhances epithelial-mesenchymal transition (EMT) through suppressing E-cadherin and regulating WNT pathway in non-small cell lung cancer (NSCLC). Biomed Pharmacother 95: 331-338, 2017.

31. Yang T, Li H, Chen T, Ren H, Shi P and Chen M: LncRNA MALAT1 depressed chemo-sensitivity of NSCLC cells through directly functioning on miR-197-3p/p120 catenin axis. Mol Cells 42: 270-283, 2019. 
32. Liu W: LncRNA LINC-PINT inhibits cancer cell proliferation, invasion, and migration in osteosarcoma by downregulating miRNA-21. Cancer Biother Radiopharm 34: 258-263, 2019.

33. Chao Y and Zhou D: IncRNA-D16366 is a potential biomarker for diagnosis and prognosis of hepatocellular carcinoma. Med Sci Monit 25: 6581-6586, 2019.

34. Jiang N, Meng X, Mi H, Chi Y, Li S, Jin Z, Tian H, He J, Shen W, Tian H, et al: Circulating lncRNA XLOC 009167 serves as a diagnostic biomarker to predict lung cancer. Clin Chim Acta 486: 26-33, 2018.

35. Xie Y, Zhang Y, Du L, Jiang X, Yan S, Duan W, Li J, Zhan Y, Wang L, Zhang S, et al: Circulating long noncoding RNA act as potential novel biomarkers for diagnosis and prognosis of non-small cell lung cancer. Mol Oncol 12: 648-658, 2018.
36. Huang SH and O'Sullivan B: Overview of the 8th edition TNM classification for head and neck cancer. Curr Treat Options Oncol 18: 40, 2017

37. Saffarzadeh AG, Blasberg JD and Beyond TNM: Searching for new patient-centric prognostic indicators in NSCLC. Ann Surg Oncol 25: 3425-3426, 2018

38. Zhou M, Zhang Z, Zhao H, Bao S, Cheng L and Sun J: An immune-related six-lncRNA signature to improve prognosis prediction of glioblastoma multiforme. Mol Neurobiol 55: 3684-3697, 2018.

(i) $\Theta$ This work is licensed under a Creative Common Attribution-NonCommercial-NoDerivatives 4.0 International (CC BY-NC-ND 4.0) License. 